\title{
Analysis of causes for late presentation of Indian patients with vestibular schwannoma
}

\author{
R Ambett, V Rupa, V RajSheKhaR*
}

\begin{abstract}
Objective: To determine the causes of delay in diagnosis and treatment of Indian patients with vestibular schwannomas.

Methods: In a prospective study from 2003 to 2005, 50 patients with a confirmed diagnosis of vestibular schwannoma were interviewed to determine the causes for (1) the delay between the patient noting the initial symptom and the definitive diagnosis, and (2) the reasons for delayed diagnosis.

Results: In 90 per cent of patients, the initial symptom was either hearing loss (62 per cent), vertigo (24 per cent) or tinnitus (4 per cent). However, most patients had been diagnosed and had presented for surgery only after neurological symptoms had became apparent. The delay between the initial medical consultation and the final diagnosis ranged from one month to 204 months (mean \pm standard deviation, $32.2 \pm 38.9$ months). After the patient had noted symptoms, the diagnosis of vestibular schwannoma was delayed due to doctor-related causes in 80 per cent of cases, and due to patient-related causes in 20 per cent. Delay following diagnosis was minimal.

Conclusions: Delay in the diagnosis of vestibular schwannoma in Indian patients is due to both doctorand patient-related factors.
\end{abstract}

Key words: Sensorineural Hearing Loss; Vestibular Schwannoma; Diagnosis; India

\section{Introduction}

Vestibular schwannoma is a benign brain tumour, and its site of origin and propensity to produce symptoms potentially make early detection and complete resection a definite possibility. In actual fact, however, many patients present late in the course of the disease with increased pre- and postoperative morbidity. ${ }^{1}$ Unilateral or asymmetrical audiovestibular symptoms were the earliest complaints in over 75-90 per cent of patients in some series. ${ }^{1-3}$ The earliest opportunity for diagnosis, therefore, lies with the ENT surgeon. Awareness of the need for screening for vestibular schwannoma in such patients should prompt appropriate referral for audiological and radiological tests.

The advent of gadolinium magnetic resonance imaging (MRI) scanning of the brain has enabled the diagnosis of tumours as small as $2 \mathrm{~mm}$. ${ }^{4}$ Yet, patients continue to present late with multiple cranial nerve palsies, cerebellar signs and features of raised intracranial pressure. In 1990, Thomsen and $\operatorname{Tos}^{3}$ lamented the fact that, despite a nationwide, intensive campaign to diagnose vestibular schwannoma at an early stage, in a small country such as Denmark with well organised referral systems, an increasing number of patients with tumours $>40 \mathrm{~mm}$ in size were being seen. They attributed this to the fact that unilateral, progressive hearing loss was still a neglected symptom, both by patients and doctors. Moffat et al. ${ }^{5}$ reported in 2004 that in spite of the easy availability of MRI scanning facilities and a good referral system in their part of the UK, they continued to see patients with giant vestibular schwannomas, with increasing frequency. They felt that many patients with large tumours had probably been asymptomatic in the early stages. In the Indian context, where resources and factual information on health and disease are limited in many parts of the country, this problem is even more pronounced. Consequently, the average size of the tumour seen in many case series from different parts of the country, including our own institution, is $>35 \mathrm{~mm}$.

In order to determine the medical and social factors responsible for late presentation of Indian patients with vestibular schwannoma, a prospective study of 50 patients with radiologically and histologically proven vestibular schwannoma was undertaken at our institution. 


\section{Materials and methods}

The study was conducted prospectively at Christian Medical College, Vellore, India, between 2003 and 2005. We included all patients who were admitted to the neurosurgical ward or who presented to the ENT out-patient clinic for evaluation and treatment of a cerebellopontine angle mass diagnosed by MRI or computed tomography (CT) scanning of the brain. Any patient who was subsequently found not to have a histological diagnosis of vestibular schwannoma was excluded from the study.

Patients were interviewed with regard to the nature and duration of their presenting symptom, delay between first symptom and first medical contact, and the type of doctor consulted initially and subsequently until definitive diagnosis and treatment. Other data recorded comprised: duration of delay between initial symptom onset and definitive diagnosis; reason for delayed diagnosis; type of misdiagnosis; and duration of delay between definitive diagnosis and treatment. Specific note was made of whether the patient used a telephone regularly. We recorded the type and results of investigations ordered prior to definitive diagnosis, namely: CT or MRI scan of the brain, pure tone audiometry (PTA), and brainstem auditory evoked response test audiometry. We also recorded the size of the tumour on contrast-enhanced MRI or CT scan of the brain in the immediate pre-operative period, measured in millimetres and categorised as $<20 \mathrm{~mm}, 20-40 \mathrm{~mm}$ and $>40 \mathrm{~mm}$.

All 50 patients included in the study underwent complete otoneurological examination and audiometric investigation. The pure tone average was calculated as the average of audiometric thresholds at $500 \mathrm{~Hz}$ and at 1,2 and $4 \mathrm{kHz}$, and was categorised as normal $(<20 \mathrm{~dB})$, mild $(25-40 \mathrm{~dB})$, moderate to severe $(45-90 \mathrm{~dB})$ and profound $(>90 \mathrm{~dB})$ hearing loss. Brainstem auditory evoked response testing was performed in those who had sufficient residual hearing.

\section{Statistical analysis}

The mean, median, mode and standard deviations were calculated for all the parameters studied. The Student $t$-test was performed to determine the significance of regular telephone use on awareness of hearing loss.

\section{Results}

\section{Patient demographics}

The study included 50 patients, 32 males (mean 41.3 years, range 15-79 years) and 18 females (mean 42.6 years, range 22-76 years). Forty-six per cent of patients were from rural areas.

\section{Initial symptoms}

Hearing loss. Hearing loss was the initial symptom in 31 (62 per cent) patients, and was the only presenting symptom in 14 ( 28 per cent) (see Table I). Audiological testing showed that 49 of 50 patients had some
TABLE I

PRESENTING SYMPTOMS IN PATIENTS* WITH VESTIBULAR SCHWANNOMA

\begin{tabular}{lc}
\hline Presenting symptom & $n(\%)$ \\
\hline Hearing loss & $31(62)$ \\
Vertigo & $12(24)$ \\
Tinnitus & $2(4)$ \\
Headache & $5(10)$ \\
\hline
\end{tabular}

Total $n=50$.

degree of hearing loss. Hearing loss was generally found to be severe ( 38 per cent) or profound ( 44 per cent) in those complaining of this symptom. The sole patient with normal hearing had vertigo alone as his presenting symptom.

Frequency of telephone use vs presence of hearing loss. We hypothesised that frequent telephone use would lead to increased awareness of hearing loss. Twenty-four patients had used the telephone regularly, of whom 17 (68 per cent) complained of hearing loss. On audiometric evaluation, most patients who used the telephone regularly (i.e. 24 patients). (83.3 per cent) had hearing loss in the affected ear, which was either severe (50 per cent) or profound ( 33.3 per cent). Of the 26 patients who did not use the telephone regularly, 14 (54 per cent) complained of hearing loss. Although a slightly higher proportion of those using the telephone complained of hearing loss, this difference was not statistically significant (chi-square test; $p=0.90$ ).

Vertigo. Vertigo was the second most common presenting symptom, with 12 (24 per cent) of the 50 patients seeking help for this. Seven of these 12 patients had seen a general practitioner and five had consulted a specialist primarily for this symptom. Specialists seen included a neurologist, a cardiologist, a physician, a gynaecologist and a general surgeon. None of those presenting with vertigo went directly to an ENT specialist. Interestingly, only five patients with vertigo as a presenting symptom were secondarily referred to an ENT specialist. However, the ENT surgeon undertook correct investigation and arrived at a diagnosis in only one of these patients.

Tinnitus. Two (4 per cent) of the 50 patients presented with an initial symptom of tinnitus. One of these two had giddiness along with tinnitus and hence ignored the latter symptom. The other patient had associated hearing loss, for which he consulted an ENT specialist, demonstrating that unilateral tinnitus is often neglected as a symptom.

Headache and other non-otological symptoms. Five (10 per cent) of the 50 patients presented initially with headache; these patients were seen initially by either a general practitioner or a neurologist and were then referred to a neurologist or neurosurgeon for definitive evaluation. Several patients presented 
with a combination of other symptoms, such as facial pain, facial weakness, limb weakness or loss of consciousness.

\section{Number and type of doctor visited prior to definitive diagnosis}

The number of doctors consulted by a patient prior to definitive diagnosis ranged from one to nine, with a mean of 2.9 doctors. Twenty-eight (56 per cent) patients had consulted a general practitioner after the onset of their initial symptom. Twenty-one patients had directly visited a specialist, of whom only 13 had consulted an ENT surgeon primarily. One patient with headache had initially consulted a traditional healer, but had subsequently sought an opinion from a neurologist, who had made the correct diagnosis.

Prior to definitive diagnosis, 26 (52 per cent) patients had seen an ENT specialist; of these 26 patients, only five (19 per cent) were correctly diagnosed as having vestibular schwannoma (Table II). Four of these five patients had a presenting symptom of hearing loss, and only one had presented with vertigo.

The type of doctor consulted, according to patients' presenting symptoms. Of the 13 patients who primarily consulted an ENT specialist, imaging was not ordered for any. Four of these patients were subsequently referred to a neurologist or neurosurgeon and were rightly diagnosed as having a cerebellopontine angle tumour, after CT scanning of the brain. Five patients who were referred on to an ENT specialist were correctly diagnosed by imaging. There was a tendency, therefore, for ENT specialists to order CT brain scans only if a patient had been referred, and not if the patient had consulted them primarily.

Pure tone audiometry was not ordered routinely for any patients seen by ENT specialists, either primarily (13 patients) or secondarily (13 patients). Only seven of the 13 patients seen primarily by an ENT specialist underwent PTA testing, despite having hearing loss as the predominant symptom. Similarly, only four of the 13 patients seen secondarily by an ENT specialist underwent PTA.

\section{Delays in diagnosis}

The reasons for delayed diagnosis were grouped into doctor-related causes (i.e. delay due to misdiagnosis or other reasons; 80 per cent) and patient-related causes (20 per cent).

Delay due to doctor-related causes. Of the total group of 50 patients, 31 presented with hearing loss, 28 of whom (56 per cent of total group) were diagnosed with idiopathic hearing loss, presbyacusis, possible ototoxicity or chronic suppurative otitis media (CSOM). Some presentations of headache and vertigo were incorrectly attributed to neurological conditions such as cervical spondylosis, migraine and intracranial haemorrhage. Some patients presenting with vertigo, vomiting and headache were misdiagnosed with dental, ophthalmological or gastrointestinal problems (see Table III).

Delay due to patient-related causes. Ten patients delayed their diagnosis by ignoring their symptoms of hearing loss $(n=4)$, tinnitus $(n=1)$, vertigo $(n=2)$ or headache $(n=1)$. One patient who was advised by a neurologist to undergo imaging did not do so due to insufficient funds. Another patient deliberately delayed undergoing imaging because of advice to the contrary from their family.

\section{General practitioner referrals}

The general practitioners' referral pattern is shown in Table IV. Only 11 (22 per cent) of the 28 patients who consulted a general practitioner initially with symptoms of hearing loss, tinnitus or vertigo were subsequently seen by ENT specialists. Most general practitioner referrals were to neurologists, neurosurgeons, ophthalmologists or other specialists. One patient with hearing loss and otitis media was not referred to an ENT specialist. Most specialist referrals were made when patients presented with such symptoms as headache, ataxia, loss of consciousness or blurred vision. Patients with vertigo who presented to a general practitioner were more likely to be referred to a neurologist or an internal medicine specialist than to an ENT specialist.

\section{Investigations}

The two commonest investigations ordered by all clinicians in this series were brain CT scan (38 patients) and PTA (17 patients). Only one patient who consulted a general practitioner initially was advised to undergo a brain CT scan. In all the

TABLE II

CLINICAL PROFILE OF PATIENTS CORRECTLY DIAGNOSED WITH VESTIBULAR SCHWANNOMA BY AN ENT SURGEON

\begin{tabular}{|c|c|c|c|c|c|}
\hline \multirow[t]{2}{*}{ Pt no } & \multicolumn{2}{|c|}{ Presenting symptom } & \multirow[t]{2}{*}{ Investigation } & \multirow[t]{2}{*}{ HL type } & \multirow[t]{2}{*}{ Tumour size $(\mathrm{mm})$} \\
\hline & Type & Duration (mths) & & & \\
\hline 1 & $\mathrm{HL}$ & 24 & PTA, MRI & Mod & 15 \\
\hline 2 & Vertigo & 36 & PTA, CT & Mod & 60 \\
\hline 3 & $\mathrm{HL}$ & 18 & PTA, CT & Prof & 32 \\
\hline 4 & $\mathrm{HL}$ & 24 & CT & Prof & 40 \\
\hline 5 & $\mathrm{HL}$ & 204 & PTA, CT & Prof & 31 \\
\hline
\end{tabular}

Pt no = patient number; mths = months; $\mathrm{HL}=$ hearing loss; PTA $=$ pure tone audiometry; $\mathrm{MRI}=$ magnetic resonance imaging; $\mathrm{CT}=$ computed tomography; Mod = moderate; Prof = profound 
TABLE III

DOCTORS' MISDIAGNOSES, BY PRESENTING SYMPTOM

\begin{tabular}{lrrrr}
\hline Misdiagnosis & Pts $(n)$ & \multicolumn{2}{c}{ Presenting symptom $(n)$} \\
\cline { 3 - 5 } & & HL & Vertigo & Tinnitus \\
\hline Idiopathic hearing loss & 16 & 15 & 0 & 1 \\
Cervical spondylosis, migraine or IC bleed & 5 & 1 & 3 & 0 \\
Presbyacusis & 5 & 4 & 0 & 0 \\
CSOM & 4 & 1 & 3 & 0 \\
Systemic illness* & 4 & 3 & 1 & 0 \\
Ototoxicity & 4 & 0 & 1 & 0 \\
Eye or tooth disorder & 3 & 0 & 0 \\
\hline
\end{tabular}

*Hypertension, diabetes mellitus, hypothyroiditis or gastrointestinal disorder. Pts = patients; HL = hearing loss; IC = intracranial; $\mathrm{CSOM}=$ chronic suppurative otitis media

other 27 patients, CT scanning was ordered only by the specialist who subsequently saw the patient. Seventeen out of the 31 patients complaining of hearing loss did not undergo PTA prior to definitive diagnosis. Only 11 of the 26 patients seen by an ENT specialist for audiovestibular symptoms underwent PTA.

Thirteen patients underwent additional MRI scanning of the brain. Only two patients underwent brainstem auditory evoked response testing prior to definitive diagnosis; one for evaluation of vertigo and the other for hearing loss. Only one patient underwent the classically prescribed order of investigations of PTA followed by BAER and diagnostic gadolinium enhanced MRI scan of the brain. Electronystagmography was performed in only one patient, who had presented with vertigo.

Most patients who complained of hearing loss as their earliest symptom were not referred for further tests until they developed ataxia $(n=5)$, headache $(n=2)$, facial nerve weakness $(n=1)$ or loss of consciousness $(n=1)$.

\section{Tumour size vs hearing loss severity}

At diagnosis, the mean tumour size was $38.9 \mathrm{~mm}$ (range, $16-70 \mathrm{~mm}$ ). At this stage, 26 (52 per cent) patients had giant tumours (i.e. $>40 \mathrm{~mm}), 20$ (40 per cent) had tumours $20-40 \mathrm{~mm}$ in size and four ( 8 per cent) had tumours $<20 \mathrm{~mm}$ in size.

There was no correlation between tumour size and hearing loss severity. Of the four patients diagnosed with tumours $<20 \mathrm{~mm}$ in size, three had presented with severe hearing loss. Conversely, two patients presenting with mild hearing loss had giant tumours.

TABLE IV

GP REFERRALS, BY PRESENTING SYMPTOM

\begin{tabular}{llllll}
\hline Symptom & MD & Neurologist & Neurosurgeon & ENT & Other \\
\hline HL & 0 & 3 & 4 & 6 & 0 \\
Vertigo & 2 & 0 & 3 & 2 & 0 \\
Tinnitus & 0 & 1 & 0 & 0 & 0 \\
Headache & 0 & 2 & 1 & 0 & $1^{*}$ \\
\hline
\end{tabular}

*The single patient with headache as a presenting symptom who was first seen by a GP and referred to another GP rather than a specialist. $\mathrm{GP}=$ general practitioner; $\mathrm{MD}=$ general physician; HL = hearing loss

\section{Delay between first consultation and final diagnosis}

The interval between the patient's first consultation and the establishment of their final diagnosis ranged from one to 204 months (mean \pm standard deviation, $32.2 \pm 38.9$ months).

\section{Delay between diagnosis and treatment}

Definitive surgery was performed within one month of diagnosis in 23 patients, within three months in 20 and within six months in five. Thus, 48 (96 per cent) patients underwent surgery within six months of definitive diagnosis. The two patients whose treatment was delayed more than six months chose this delay themselves, one due to fear of surgery and the other due to a desire to try homeopathy first.

\section{Discussion}

There are three possible sources for a delay in the diagnosis and treatment of a patient with vestibular schwannoma. Firstly, the patient may not notice their initial symptom (e.g. unilateral hearing loss) or, having noticed it, may not attribute any significance to it. Secondly, the patient might seek medical attention for their symptom, but the attending doctor may not correctly pursue the cause of the symptom. Thirdly, having consulted a doctor and been advised to undergo appropriate investigations, the patient may not pursue the advised course, due to lack of funds (when subsidised care is not readily available) or fear of possible complications of treatment. In our study, the first two causes were responsible for the delay in diagnosis and management in the vast majority of patients.

\section{Patient-related diagnostic delay}

It is difficult, if not impossible, to quantify the delay in vestibular schwannoma diagnosis attributable to the patient's neglect of their symptoms (usually unilateral hearing loss or tinnitus). This is because most patients are unaware of when their earliest symptoms began. Several patients first became aware of hearing loss only after audiological testing revealed a deficit. In our series, hearing loss was the most common symptom at presentation; furthermore, up to 82 per cent of patients had severe or profound 
hearing loss at the time of diagnosis. Patients' lack of awareness of unilateral hearing loss may be related to the fact that they live or work in areas of high ambient noise levels. More often, perhaps, for either financial or social reasons, an individual might not want to draw attention to this seemingly innocuous symptom. The importance of public awareness of the significance of progressive, asymmetrical audiovestibular symptoms cannot be overstated. In developing countries such as India, this is just one of many health education topics that require emphasis. However, such awareness is a problem even in developed countries, as seen in reports from Denmark, ${ }^{3}$ the $\mathrm{USA}^{6}$ and the Netherlands.

In the USA, Rosenberg ${ }^{8}$ found that the mean delay in vestibular schwannoma diagnosis in patients treated non-surgically was 7.3 years. In Denmark, Thomsen and Tos $^{3}$ reported a mean delay of 7.1 years after onset of initial symptoms; an overall delay of 6.9 to 7.1 years, due to either patient- or otologist-related factors, occurred in over 70 per cent of patients. These authors emphasised the need for increased public and physician awareness. Seven years later in Denmark, following increased availability of scanning facilities and improved patient and physician awareness, this same research group reported that the diagnostic delay had reduced, to one to five years in 43 per cent of patients, being more than five years in only 32 per cent. ${ }^{9}$

Could some tumours remain asymptomatic until they reach a considerable size? Moffat et $a l^{5}$ were of this opinion, after noting an increasing number of large vestibular schwannomas over a 10-year period, despite a sound referral system and easily accessible MRI scanning facilities. In both developing and developed countries, a small percentage of patients present late with few audiovestibular symptoms or have an atypical presentation, thus eluding early diagnosis.

A recent, systematic review of observational studies of patients with vestibular schwannoma who were managed conservatively found variable tumour growth, ranging from 0.4 to $2.9 \mathrm{~mm} /$ year, with an average growth rate of $1.2 \mathrm{~mm} /$ year. $^{10}$ If the highest rate of tumour growth were assumed, a patient with a $30 \mathrm{~mm}$ vestibular schwannoma would have harboured the tumour for nearly 10 years. Therefore, in our series, the major delay in diagnosis was caused by patients' own lack of awareness, or concern, regarding their presenting symptom, usually unilateral hearing loss.

\section{Doctor-related diagnostic delay}

In our series, patients with vestibular schwannoma who presented with hearing loss to a doctor were often misdiagnosed. Misdiagnosis was more often seen in middle-aged or older patients with hearing loss or mild symptoms of imbalance. The presence of coexistent disease, such as CSOM or hypertension, was a confounding factor in many patients. Van Leeuwen et al. ${ }^{7}$ found similarly that such misdiagnoses as familial hearing loss, Ménière's disease, otosclerosis and alcoholism were made in 27 of 164 patients with asymmetrical audiovestibular symptoms who were later proven to have vestibular schwannoma. Thomsen and $\operatorname{Tos}^{3}$ reported that, even in a developed country such as Denmark, some patients presenting with hearing loss to an otologist did not undergo PTA. A low index of suspicion was the most likely reason. In India, a paucity of hearing assessment facilities and of trained audiometrists and audiologists makes it difficult for general practitioners and specialists to order PTA in patients complaining of hearing loss. Evidently, increased awareness should go hand in hand with the provision of easily accessible audiological and imaging facilities.

- Vestibular schwannoma diagnosis is frequently delayed, for a variety of reasons. These reasons have not been studied in patients in the developing world (e.g. India)

- This prospective study of $\mathbf{5 0}$ patients with vestibular schwannoma found that such diagnostic delay was caused both by patient behavioural factors and by doctors' lack of awareness of vestibular schwannoma

- Although the mean delay from patient presentation to correct diagnosis was only three years, the large tumour size at diagnosis indicated that most patients had probably been unaware or unconcerned regarding unilateral hearing loss, for several years before presentation

- Increased awareness of vestibular schwannoma as a cause of unilateral audiovestibular symptoms, amongst general practitioners and otolaryngologists, may reduce the delay in vestibular schwannoma diagnosis

In the Indian context, the system of medical practice and patterns of referral are also important factors contributing to delays in vestibular schwannoma diagnosis. Essentially, there is no well defined pattern of referral. Specialists often willingly accept and treat patients with problems far removed from their area of specialisation. This was evident from our study findings; cardiologists and general surgeons managed several patients with audiological and neurological complaints. The lack of a proper referral hierarchy makes it difficult to reduce the risk of misdiagnosis when a patient with hearing loss does present early to a physician. It is interesting to note that, in our series, patients were evaluated by an average of over three doctors (range, one to nine) before a diagnosis was made.

In the present study, only 17 patients underwent PTA before diagnosis, despite the fact that 62 per cent of patients complained of hearing loss. Correlating the initial symptom with the symptom that prompted referral, it was found that when patients presented with hearing loss to a general practitioner, 
it was not thought necessary to refer the patient to an ENT specialist. In all but three patients, referral was considered only when more sinister symptoms developed, such as headache, ataxia or loss of consciousness. Most patients were diagnosed with brain CT scans performed in or close to their home town, suggesting that facilities for CT scanning are more widely available than those for audiological testing. In several states of the country, public hospitals provide CT scanning facilities at a nominal cost, making this a far more accessible facility than PTA.

Our study showed that, once a diagnosis of vestibular schwannoma was established, the patient and doctor generally did not delay surgery; this was the case for 96 per cent of our patients. This is in sharp contrast to the experience in the Netherlands, where the average delay between diagnosis and surgery (termed 'specialist delay') for 164 patients was 15.2 months. ${ }^{7}$ Non-surgical management of vestibular schwannoma includes a 'watch and wait' policy and radiosurgery treatment. Both these modes of management apply to patients with very small tumours, which were not encountered in our series. The high rate of surgical intervention found in our series was a reflection of the large size of the tumours found.

\section{Prevalence of large vestibular schwannoma}

The relative prevalence of large vestibular schwannoma indirectly reflects the delay in diagnosis. Of 1500 US patients with vestibular schwannoma, 39.5 per cent had tumours larger than $2.5 \mathrm{~cm} ;{ }^{11}$ there was no further categorisation on the basis of size in this series. In a series of 1000 vestibular schwannomas reported from Hanover, Germany, ${ }^{12} 36$ per cent of patients had giant tumours $(>40 \mathrm{~mm})$ while 44 per cent had tumours between 30 and $40 \mathrm{~mm}$ in size; therefore, nearly 80 per cent of patients had large tumours measuring $>30 \mathrm{~mm}$. In a recent series of 259 Indian patients with vestibular schwannoma, ${ }^{13} 56$ per cent had giant tumours and another 41 per cent had tumours measuring 25 to $40 \mathrm{~mm}$ in size; thus, 97 per cent of patients had tumours measuring over $25 \mathrm{~mm}$ in size. Although these three series are not strictly comparable due to the differing reporting methodologies used, they do indicate that large tumours constitute a substantial proportion, if not the majority, of vestibular schwannomas seen worldwide, except probably in the USA ${ }^{11}$ and Denmark, ${ }^{9}$ where most patients currently seem to have smaller tumours at diagnosis. This suggests that presentation of vestibular schwannoma is unduly delayed, even in developed countries. This is probably a reflection of poor patient awareness of unilateral hearing loss.

\section{Strategies for early diagnosis of vestibular schwannoma}

The ideal strategy for early vestibular schwannoma detection would be patient education regarding the need to seek investigation and treatment for unilateral hearing loss. However, this is unlikely to succeed, as some patients are not even aware of their hearing loss until it is brought to their attention during testing. In the light of our findings, it appears that a more feasible strategy for the early diagnosis of vestibular schwannoma would comprise educating doctors, specifically general practitioners and ENT specialists, about the need for intensive investigation of a patient with unilateral audiovestibular symptoms. The use of either audiological screening protocols followed by imaging ${ }^{14,15}$ or direct referrals for gadolinium MRI brain scanning, ${ }^{16,17}$ depending on the resources available in a particular setting, would ensure that most cases were not missed.

\section{Conclusions}

In our patients, the average delay in vestibular schwannoma diagnosis was about three years; this is similar to findings from developed countries. However, the average size of our patients' tumours was much larger. This suggests the need for greater patient and physician awareness of the importance of detailed evaluation of unilateral audiovestibular symptoms.

\section{References}

1 Moffat DA, Hardy DG, Baguley DM. Strategy and benefits of acoustic neuroma searching. J Laryngol Otol 1989;103: $51-9$

2 Haapaniemi J, Laurikainen E, Johansson R, Miettinen S, Varpula M. Cochleovestibular symptoms related to the site of vestibular schwannoma. Acta Otolaryngol Suppl 2000;543:14-16

3 Thomsen J, Tos M. Acoustic neuroma. Clinical aspects, audiovestibular assessment, diagnostic delay and growth rate. Am J Otol 1990;11:12-19

4 Stack JP, Ramsden RT, Antoni NM, Lye RH, Isherwood I, Jenkins JP. MRI imaging of acoustic neuromas: the role of gadolinium DTPA. Br J Radiol 1988;61:800-5

5 Moffat DA, Jones SE, Mahendran S, Humphriss R, Baguley DM. Referral patterns in vestibular schwannomas - 10 years on. Clin Otolaryngol Allied Sci 2004;29: 515-17

6 Traquina DN, Guttenberg I, Sasaki CT. Delayed diagnosis and treatment of acoustic neuroma. Laryngoscope 1989;99: 814-18

7 van Leeuwen JPPM, Harhangi BS, Thewissen NPMW, Thijssen HOM, Cremers CWRJ. Delays in the diagnosis of acoustic neuromas. Am J Otol 1996;17:321-5

8 Rosenberg SI. Natural history of acoustic neuromas. Laryngoscope. 2000;110:497-508

9 Tos M, Charabi S, Thomsen J. Increase of diagnosed vestibular schwannomas in Denmark. Acta Otolaryngol Suppl 1997;529:53-5

10 Yoshimoto Y. Systematic review of the natural history of vestibular schwannoma. J Neurosurg 2005;103: $59-63$

11 Ryzenman JM, Pensak ML, Tew JM Jr. Headache: a quality of life analysis in a cohort of 1657 patients undergoing acoustic neuroma surgery, results from the acoustic neuroma association. Laryngoscope 2005;115: $703-11$

12 Samii M, Matthies C. Management of 1000 vestibular schwannomas (acoustic neuromas): the facial nervepreservation and restitution of function. Neurosurgery 1997; 40:684-94

13 Jain VK, Mehrotra N, Sahu RN, Behari S, Banerji D, Chhabra DK. Surgery of vestibular schwannomas: an institutional experience. Neurol India 2005;53:41-5 
14 Rupa V, Job A, George M, Rajshekhar V. Cost-effective screening of vestibular schwannoma: ABR or MRI? Otolaryngol Head Neck Surg 2003;128:823-8

15 Welling DB, Glasscock ME, Woods CI, Jackson CG Acoustic neuroma: a cost-effective approach. Otolaryngol Head Neck Surg 1990;103:364-70

16 Robson AK, Leighton SE, Anslow P, Milford CA MRI as a single screening procedure for acoustic neuroma: a cost effective protocol. J R Soc Med 1993;86: $455-7$

17 Ravi KV, Wells SC. A cost effective screening protocol for acoustic neuroma screening in the late 1990s. J Laryngol Otol 1996;110:1129-32
Address for correspondence:

Dr V Rupa,

Department of ENT,

Christian Medical College,

Vellore 632 004, India.

Fax: 0914162232103

E-mail: rupavedantam@cmcvellore.ac.in

Dr V Rupa takes responsibility for the integrity of the content of the paper.

Competing interests: None declared 